Postgrad. Med. J. (1965), 41, 469

\title{
CYSTECTOMY IN THE MANAGEMENT
}

\section{OF CARCINOMA OF THE URINARY BLADDER}

\author{
Justin J. CORDONNIER, M.D.
}

From the Department of Surgery, Section on Urology, Washington University School of Medicine, St. Louis, Missouri.

SIMPLE cystectomy has proved to be a satisfactory method of treatment for carcinoma of the urinary bladder. We became interested in this method of treatment in 1948 and have performed 265 cystectomies since then. During the period between 1948 and 1953, ureterosigmoidostomy was utilized as a means of urinary diversion. Since then, we have been using the ileal loop as a method of urinary diversion. In our opinion, the morbidity has been decreased and the prognosis for long term survival is definitely enhanced because of improvement in the method of urinary diversion.

In our original series reported in 1956, a total of 48 five year cases was analyzed. In this group, there were 2 operative deaths, an operative mortality of $4.2 \%$. Thirty patients died within the five-year period and sixteen survived five or more years, a survival rate of $33.5 \%$. Incorporated in this series was a large number of patients operated upon early in our experience. The criteria for surgical removal were not well formulated, and cystectomy was performed in many cases in whom there was no possibility of ultimate cure. With additional experience, we have developed more rigid indications for cystectomy, resulting in an improvement in five-year survival rates.

Cystectomy is now performed on patients in the following categories: lesions of high grade cellular malignancy (Grade III and IV); biopsy evidence of muscle invasion; rapid recurrence of tumours of increasing cellular malignancy; and generalized involvement of the bladder mucosa by tumour, not controllable by other means.

Cystectomy has not been performed in those patients in whom, on abdominal exploration, the following conditions were found: extravesical spread of tumour; the presence of positive pelvic lymph nodes; the presence of metastatic disease outside the pelvis.

The presence of palpable lymph nodes, demonstrated to contain carcinoma by frozen section, is definitely an indication for palliative diversion without any further surgery on the bladder. In our experience, patients with positive lymph nodes do not survive beyond a year. We do not feel that there is any particular advantage in routinely combining radical lymph node dissection with the cystectomy. The lymph node dissection seems to add materially to both the mortality and morbidity of the surgery; and, since the prognosis is extremely poor if positive nodes are present, it is our belief that the rare case who might be salvaged by this method is more than offset by the added mortality and morbidity involved.

A recent review of 99 patients followed five or more years after cystectomy and ileal loop diversion showed an overall survival rate of $40(41.4 \%)$. The immediate operative mortality was $4.2 \%$. An analysis of this group based on both Broder's classification and Jewett's classification is of interest.

TABLE 1

Five Year Survival BRoder's Classification

\begin{tabular}{lllll}
\hline Grade & I & II & III & IV \\
Died & 3 & 14 & 27 & 14 \\
5 Year Survivors & 1 & 13 & 17 & 10 \\
\hline Total & 4 & 27 & 44 & 24 \\
\hline$\% 5$ Year Survival & 25 & 48.2 & 38.6 & 41.7 \\
\hline
\end{tabular}

TABLE 2

Five Year Survival

JEWETT Classification

\begin{tabular}{lrrrrr}
\hline & $\mathbf{A}$ & $\mathbf{B}_{1}$ & $\mathbf{B}_{2}$ & \multicolumn{1}{c}{$\mathbf{C}$} & D \\
\hline Died & 20 & 8 & 6 & 16 & 8 \\
\hline 5Year Survivors & 22 & 8 & 4 & 7 & 0 \\
\hline Total & 42 & 16 & 10 & 23 & 8 \\
\hline$\% 5$ Year Survival & 52.4 & 50 & 40 & 30.4 & 0
\end{tabular}


It will be noted that although cystectomy was rarely performed for the Grade I lesion, the five-year survival in this group was rather poor. This probably indicates that the disease was rather far advanced in patients of this category on whom cystectomy was performed. It also is indicative of the fact that even Grade I tumours can be fatally malignant in the advanced stages. Five year survival of the Broder's Grades II, III, and IV shows little variation.

It is our impression that the Broder's classification alone has limited prognostic value and that Jewett's classification is of far more significance clinically as regards management and prognosis. In our experience, accurate determination of depth of penetration into the muscle pre-operatively has been extremely difficult. Therefore, we have adopted the principle that any degree of muscle penetration found on biopsy is an indication for cystectomy. This finding would seem to signify either a tumour of fairly high potential malignancy of relatively short duration; or, if of lower biologic potential, of long enough standing to warrant a radical approach.

As one would expect, the highest five-year survival group are those with the more superficial lesions. However, interestingly enough, there are a significant number of five year survivals in the Jewett $\mathrm{C}$ group. To me, this would indicate that each patient warrants surgical exploration and removal of the bladder if technically feasible.

In the more advanced cases, where the probability of cure was limited, we have been impressed by the degree of palliation obtained by removal of the bladder even though the patient eventually died of carcinoma. Therefore, the contra-indications have not been too rigidly observed and if, in our opinion, the bladder is removable, although there is a suggestion of local fixation, we are inclined to proceed with the cystectomy.

Supervoltage radiation, particularly in the $\frac{c}{.}$ post-operative period, has added very little to $\overrightarrow{\overrightarrow{\mathrm{S}}}$ the overall five-year survival, and in some instances has actually created almost insurmountable problems. Three patients of our $\frac{\bar{c}}{\mathrm{~m}}$ series, who received substantial post-operative $\frac{\bar{\phi}}{\overrightarrow{ }}$ supervoltage radiation, developed gastro- $\unrhd$ intestinal complications of such magnitude that, के although as far as can be determined they are $\overrightarrow{0}$ free of carcinoma for a prolonged period of time, they have required innumerable gastro- $\vec{\omega}$ intestinal operations and are, in essence, $\frac{\mathscr{\sigma}}{0}$ abdominal cripples. Presently, we employ윽 post-operative radiation in only a limited number of patients and with extreme caution.

\section{Conclusion}

After fifteen years' experience with tatal $\overrightarrow{8}$

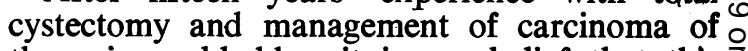
the urinary bladder, it is my belief that this procedure is of definite value in the management of bladder cancer. In a series of 990 patients followed five or more years with ileal $\frac{c}{5}$ loop diversion, there was a five-year survival $\vec{A}$ of $41.4 \%$. At risk studies on additional case performed subsequently, suggest that although or the figure may be lowered slightly, the five-yeaf survival in the future will continue to be at very satisfactory level. With the advent of ileal loop diversion and the improved long term prognosis it supplies, it is our belief that $\stackrel{\AA}{\AA}$ cystectomy should be performed more fre- $\overrightarrow{\overrightarrow{\vec{P}}}$ quently. Certainly, one would decry the use $\frac{3}{3}$ of cystectomy as a last resort when all other forms of treatment have failed. Since depth of penetration is to a certain extent directly proportional to the longevity of the disease, 3 . an earlier, more radical approach in the management of carcinoma of the bladder would $\underline{3}$. seem clearly indicated. 